\title{
Storage Stability of Chicken Momo at Super Chilling Temperature
}

\author{
A.A. Pawade, K.S. Rathod* and R.K. Ambadkar \\ Department of Livestock Products Technology, Nagpur Veterinary College, \\ Nagpur (M.S.), India \\ *Corresponding author
}

\section{Keywords}

Chicken momos, Superchilling, Aerobic packaging, Quality analysis, Shelf life

\section{Article Info}

Accepted:

24 November 2018

Available Online:

10 December 2018

\section{A B S T R A C T}

The present study was conducted to compare the quality of superchilled $\left(-2 \pm 0.5^{\circ} \mathrm{C}\right)$, frozen $\left(-20 \pm 1^{\circ} \mathrm{C}\right)$ and chilled $\left(4 \pm 1^{\circ} \mathrm{C}\right)$ chicken momos, aerobically packaged in LDPE pouches. The products stored at chilling $\left(4 \pm 1^{\circ} \mathrm{C}\right)$ temperature spoiled on day 12 . There was significant decrease in moisture and fat content of both frozen and superchilled samples with progress in storage period where as the protein content of chicken momo did not affect. The $\mathrm{pH}$ of all the samples increased gradually during the storage period. However, the $\mathrm{pH}$ of superchilled samples increased significantly at the end of storage. TBA and Tyrosine values of the superchilled samples were significantly lower than chilled samples during the storage period. Furthermore, superchilled products showed significantly lower TPC and PC as compared to chilled product and none of the samples revealed coliforms throughout the period of storage. Superchilled samples had the yeast and molds on day 24 onwards.However, frozen sample did not reveal any yeast and mold throughout the study period of 32 days. Sensory evaluation also revealed significantly high scores for superchilled product than chilled momos indicating usefulness of superchilling in extension of shelf life. The study indicated that the shelf life of chicken momos could be extended up to 32 days under superchilling $\left(-2 \pm 0.5^{\circ} \mathrm{C}\right)$ temperature.

\section{Introduction}

Due to rapidly increasing economy, population, industrialization and urbanization, there is a fast development of meat industries over recent decades. Processed, convenient and value added meat products sector particularly snack industry has very good opportunities in developed and developing countries like India due to changing consumer lifestyle, working women trends, increase in income of families, singles/professionals staying away from home, nuclear families.
Snacks are available in variety of packaged as well as processed foods like Chicken chips (Sharma and Nanda, 2002), chicken meat rings (Mishra et al., 2015), fish curls (Raja et al., 2014), momos (Tanuja et al., 2014), Chakali (Hiwarkar, 2017) etc. Momos (type of dumpling), native to Tibet and was brought to Nepal by Newari traders of Kathmandu before 1930s, are famous street food throughout the world. It is very popular in the Himalayan states of India especially in Sikkim and Ladakh. They were introduced from Han China, as one of the most popular fast food. 
There are two types of momos fried and steamed momos. Momos that are pan fried after steaming are known as kothey momo and steamed momo served in hot sauce are referred C-momo (Tanuja, 2013).

The storage temperature of chilled fresh foods is normally 4 to $10^{\circ} \mathrm{C}$ and in the distribution chain the storage temperatures also vary a lot (Hemmingsen, 2002). Most of the foods are perishable and require refrigeration in order to achieve an acceptable length of shelf life and to minimise the risk of food borne diseases. The ability to supply sufficient amounts of fresh food to a growing population is currently a major worldwide challenge. In this context, superchilling is considered as the best technology for ensuring a greater exploitation of fresh food. Superchilling is a method for conserving foods by holding the product at a temperature between -0.5 to $-4^{\circ} \mathrm{C}$ (Fennema, 1973) and results in better quality compared to conventional chilling (Einarsson, 1988) for many food products. The ice stored in superchilled products will protect from temperature rises in poor cold chains (Magnussen et al., 2008). However, most of the studies on superchilling has been conducted on seafoods (Kaale et al., 2013; Erikson et al., 2011; Olafsdottir et al., 2006; Sivertsvik et al., 2002 and 2003; Zeng et al., 2005, Duun and Rustad, 2007, 2008;) and fresh meats (Rathod et al., 2017a and Rathod et al., 2017b).

Since, scanty literature on superchilling of meat products like pork roast (Duun et al, 2008), Chicken nuggets (Kanle, 2017) is available, the present study was undertaken to investigate the effects of superchilling temperature on storage stability of chicken momo.

\section{Materials and Methods}

Fresh boneless meat from chicken, slaughtered by traditional halal method was procured from the local market. All the body fat, tendons and separable connective tissue were trimmed off from the breast fillets. These fillets were then packaged in LDPE bags, kept at refrigeration temperature $\left(4 \pm 1{ }^{0} \mathrm{C}\right)$ overnight $(12 \mathrm{~h})$ and subsequently used for product preparation.

Boneless chilled meat was cut into small pieces and minced in a mixer. Firstly salt $(1.6 \%)$ and MSG (0.4\%) were added in minced meat and mixed for 2 min with addition of condiments (10\%) and spice mix (2\%) and mixed for $2 \mathrm{~min}$. Then vegetable oil (5.5\%) along with chopped onion $(15 \%)$ and green coriander $(5 \%)$ were added and mixed properly. Chicken momos were prepared from momo mix according to the method of Vanramhlimpuii (2015) with slight modifications. The dough was prepared by proper kneading of refined wheat flour $(95.5 \%)$, refined oil (4\%), salt $(0.5 \%)$ and lukewarm water as per requirement in a bowl. $5 \mathrm{~g}$ of dough was taken, rolled in a round shape and filled with $15 \mathrm{~g}$ of momo mix and then the edges were closed properly. The chicken momo were shaped manually at ambient temperature followed by cooking in steamer for $30 \mathrm{~min}$.

The product was evaluated on the basis of the physico-chemical properties viz., $\mathrm{pH}$, TBA number, tyrosine value and microbiological analysis viz., total plate count, psychrophilic plate count, coliform count and yeast and mold count as well as sensory scores at an interval of 4 days during the study period of 32 days. The $\mathrm{pH}$, moisture (\%), fat $(\%)$ and protein $(\%)$ content of chicken nuggets were determined as per the method of AOAC (2012). Thiobarbituric acid number and tyrosine value of chicken momo samples were determined as per the method suggested by Witte et al., (1970) and strange et al.,(1977) with slight modifications. The microbiological quality of chicken momo was assessed on the basis of total plate count (TPC), psychrophilic 
count (PC),coliform count and yeast and mold count as per the procedure of APHA (1984).

The sensory evaluation of steamed chicken momos were conducted by semi-trained sensory panelists and the product was evaluated on the basis of various sensory attributes viz., appearance, flavor, texture, juiciness and overall acceptability by using 8 point Hedonic scale (Keeton et al., 1983) and the data generated was analyzed by Analysis of Variance following the procedure described by Snedecor and Cochran (1989).

\section{Results and Discussion}

\section{Physicochemical properties}

The moisture content of superchilled sample was significantly low during the entire storage period of 32 days than frozen samples. However, the moisture of both the samples decreased significantly with progress in storage period. This reduction in moisture content might be co-related with the decrease in $\mathrm{pH}$ (Huff- Lonergan and Lonergan, 2005) during storage. The results Corroborated with the findings of Rathod (2017) who reported significant reduction in moisture of frozen ($\left.20 \pm 1^{\circ} \mathrm{C}\right)$ and superchilled $\left(-1.5\right.$ to $\left.-2.5^{\circ} \mathrm{C}\right)$ chicken breast samples throughout storage period of 20 days under aerobic conditions. Similar observations were also reported by Kanle (2017) in aerobically packed superchilled chicken nuggets stored at two different superchilling $\left(-2 \pm 0.5^{\circ} \mathrm{C}\right.$ and $0.5 \pm 0.5^{\circ} \mathrm{C}$ ) temperatures.

Storage temperatures did not affect the protein content of chicken momo. There was marginal reduction in protein content of chicken momos stored at different temperatures during storage periods. Nevertheless the protein content of superchilled sample $(17.43 \pm 0.06)$ was slightly lower than that of the frozen sample (17.66 \pm 0.07). Similar results were also reported by
Tanuja (2013) who observed non significant differences in protein content of chicken momos stored at chilling temperature. Similarly, there was no significant variation in the fat content of samples between 0 and 4 days of storage. However, significant variations in the fat content of the products were observed between superchilled and frozen samples at the end of storage. The decrease in fat content during frozen storage at the end of storage might be due to lipid oxidation caused by loss of triglyceride fraction during frozen storage whereas intermediately activities of endogenous enzymes also resulted in disintegration of meat lipids (Agnihotri, 1988). Similar results were also reported by Kanle (2017) in superchilled nuggets.

The $\mathrm{pH}$ of sample differed significantly between the superchilled and frozen samples. The $\mathrm{pH}$ of all the samples increased gradually during the storage period and was significantly higher in chilled sample $(6.31 \pm 0.01)$ than

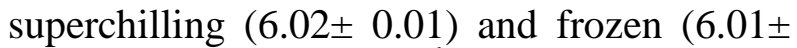
$0.01)$ samples on $12^{\text {th }}$ day of storage. However, superchilled samples showed significant increase in $\mathrm{pH}$ than frozen samples at the end of storage. This consistent increase in $\mathrm{pH}$ during storage under superchilling temperature could be due to liberation of protein metabolites by bacterial enzymes, during autolysis (Strange et al., 1977) and microbial spoilage (Dainty et al., 1975) of meat.

The TBA values of chicken momos increased significantly $(\mathrm{P}<0.05)$ from day 8 of the storage period irrespective of treatments. TBA values of different treatments on day 4 indicated significant increase in TBA value of chilled product while there was no significant variation of TBA values of superchilled and frozen product. This pattern was observed upto day 12 where the chilled samples were unacceptable sensorily (Table 1). 
Table.1 Physico-chemical changes in aerobically packed superchilled Chicken momos during storage

\begin{tabular}{|c|c|c|c|c|c|c|c|c|c|c|}
\hline \multirow{3}{*}{ Treatment } & \multicolumn{9}{|c|}{ Storage period (Days) } & \multirow{2}{*}{$\begin{array}{c}\text { Treatmen } \\
\text { t mean }\end{array}$} \\
\hline & 0 & 4 & 8 & 12 & 16 & 20 & 24 & 28 & 32 & \\
\hline & \multicolumn{9}{|c|}{ Moisture(\%) } & \\
\hline $\mathbf{T}_{1}$ & $\begin{array}{c}62.67 \pm \\
0.30_{\mathrm{a}}\end{array}$ & $\begin{array}{l}{ }^{\mathrm{A}} 61.70 \pm \\
0.26 \mathrm{~b}\end{array}$ & $\begin{array}{c}\mathrm{A} 60.85 \pm \\
0.18_{\mathrm{c}}\end{array}$ & $\begin{array}{c}{ }^{\mathrm{A}} 59.18 \pm \\
0.16_{d}\end{array}$ & $\begin{array}{l}\mathrm{A}^{\mathrm{A}} 58.04 \pm \\
0.41_{\text {ef }}\end{array}$ & $\begin{array}{c}{ }^{\mathrm{A}_{58.37}} \pm \\
0.13_{\mathrm{f}}\end{array}$ & $\begin{array}{c}{ }^{\mathrm{A}} 55.89 \pm \\
0.31_{\mathrm{g}}\end{array}$ & $\begin{array}{l}{ }^{\mathrm{A}} 54.53 \pm \\
0.28_{\mathrm{hi}}\end{array}$ & $\begin{array}{c}{ }^{\mathrm{A}} 53.91 \pm \\
0.14_{\mathrm{i}}\end{array}$ & $\begin{array}{c}\mathrm{A}_{58.35 \pm} \\
0.40\end{array}$ \\
\hline $\mathrm{T}_{2}$ & $\begin{array}{c}62.67 \pm \\
0.30_{a}\end{array}$ & $\begin{array}{l}{ }^{\mathrm{B}} 60.59 \pm \\
0.33_{\mathrm{b}}\end{array}$ & $\begin{array}{c}\text { B } 55.76 \pm \\
0.57 \mathrm{c}\end{array}$ & $\begin{array}{c}\text { B } 52.67 \pm \\
0.18_{d}\end{array}$ & Spoiled & - & - & - & - & - \\
\hline $\mathbf{T}_{3}$ & $\begin{array}{c}62.67 \pm \\
0.30_{a}\end{array}$ & $\begin{array}{c}\mathrm{C}_{62.43 \pm} \\
0.14_{\mathrm{a}}\end{array}$ & $\begin{array}{c}{ }^{\mathrm{C}} 62.07 \pm \\
0.22 \mathrm{a}\end{array}$ & $\begin{array}{c}{ }^{\mathrm{C}} 60.74 \pm \\
0.14_{\mathrm{b}}\end{array}$ & $\begin{array}{l}\mathrm{B} 60.43 \pm \\
0.11_{\mathrm{bc}}\end{array}$ & $\begin{array}{c}{ }^{\mathrm{B}} 59.12 \pm \\
0.17_{\mathrm{d}}\end{array}$ & $\begin{array}{c}{ }^{\mathrm{B}} 59.10 \pm \\
0.22_{\mathrm{de}}\end{array}$ & $\begin{array}{c}{ }^{\mathrm{B}} 58.22 \pm \\
0.16_{\mathrm{f}}\end{array}$ & $\begin{array}{c}{ }^{\mathrm{B}} 57.74 \pm \\
0.26_{\mathrm{fg}}\end{array}$ & $\begin{array}{c}\mathrm{B} 60.28 \pm \\
0.25\end{array}$ \\
\hline Days mean & $\begin{array}{c}62.67 \pm \\
0.17 \mathrm{a}\end{array}$ & $\begin{array}{c}61.57 \pm \\
0.23_{\mathrm{b}}\end{array}$ & $\begin{array}{c}59.56 \pm \\
0.69_{\mathrm{c}}\end{array}$ & $\begin{array}{c}57.53 \pm \\
0.85_{\mathrm{d}}\end{array}$ & - & - & - & - & - & \\
\hline \multicolumn{11}{|c|}{ Protein $(\%)$} \\
\hline$T_{1}$ & $\begin{array}{c}17.94 \pm \\
0.20_{a}\end{array}$ & $\begin{array}{c}17.88 \pm \\
0.06_{\mathrm{ac}}\end{array}$ & $\begin{array}{c}17.84 \pm \\
0.05_{\mathrm{ac}}\end{array}$ & $\begin{array}{c}17.80 \pm \\
0.03_{\mathrm{ab}}\end{array}$ & $\begin{array}{l}17.79 \pm \\
0.10 a_{b}\end{array}$ & $\begin{array}{c}17.6 \pm \\
0.10_{\text {bcd }}\end{array}$ & $\begin{array}{l}17.60 \pm \\
0.08_{\text {bcd }}\end{array}$ & $\begin{array}{c}17.48 \pm \\
0.05_{\mathrm{d}}\end{array}$ & $\begin{array}{c}17.43 \pm \\
0.06_{\mathrm{d}}\end{array}$ & $\begin{array}{c}17.71 \pm \\
0.04\end{array}$ \\
\hline$\overline{T_{2}}$ & $\begin{array}{c}17.94 \pm \\
0.20_{a}\end{array}$ & $\begin{array}{c}17.78 \pm \\
0.25_{\mathrm{ab}}\end{array}$ & $\begin{array}{c}17.65 \pm \\
0.16_{a}\end{array}$ & $\begin{array}{c}17.55 \pm \\
0.11_{\mathrm{b}}\end{array}$ & Spoiled & - & - & - & - & - \\
\hline $\mathbf{T}_{3}$ & $\begin{array}{c}17.94 \pm \\
0.20\end{array}$ & $\begin{array}{c}17.91 \pm \\
0.12\end{array}$ & $\begin{array}{c}17.86 \pm \\
0.04\end{array}$ & $\begin{array}{c}17.82 \pm \\
0.06\end{array}$ & $\begin{array}{c}17.79 \pm \\
0.06\end{array}$ & $\begin{array}{c}17.75 \pm \\
0.06\end{array}$ & $\begin{array}{c}17.73 \pm \\
0.09\end{array}$ & $\begin{array}{c}17.70 \pm \\
0.09\end{array}$ & $\begin{array}{c}17.66 \pm \\
0.07\end{array}$ & $\begin{array}{c}17.80 \pm \\
0.03\end{array}$ \\
\hline Days mean & $\begin{array}{c}17.94 \pm \\
0.11\end{array}$ & $\begin{array}{c}17.86 \pm \\
0.09\end{array}$ & $\begin{array}{c}17.78 \pm \\
0.06\end{array}$ & $\begin{array}{c}17.72 \pm \\
0.05\end{array}$ & - & - & - & - & - & \\
\hline \multicolumn{11}{|c|}{ Fat $(\%)$} \\
\hline $\mathbf{T}_{1}$ & $\begin{array}{c}10.66 \pm \\
0.01\end{array}$ & $\begin{array}{c}10.65 \pm \\
0.01\end{array}$ & $\begin{array}{c}{ }^{\mathrm{A}} 10.65 \pm \\
0.01\end{array}$ & $\begin{array}{c}\mathrm{AB} 10.63 \pm \\
0.01\end{array}$ & $\begin{array}{c}10.64 \pm \\
0.01\end{array}$ & $\begin{array}{c}\mathrm{A} 10.64 \pm \\
0.01\end{array}$ & $\begin{array}{c}\mathrm{A} 10.65 \pm \\
0.01\end{array}$ & $\begin{array}{c}{ }^{\mathrm{A}} 10.65 \pm \\
0.01\end{array}$ & $\begin{array}{c}\mathrm{A} 10.65 \pm \\
0.01\end{array}$ & $\begin{array}{c}\mathrm{A} 10.65 \pm \\
0.01\end{array}$ \\
\hline $\mathbf{T}_{2}$ & $\begin{array}{c}10.66 \pm \\
0.01_{\mathrm{a}}\end{array}$ & $\begin{array}{c}10.63 \pm \\
0.02_{\mathrm{a}}\end{array}$ & $\begin{array}{c}\mathrm{B} 10.60 \pm \\
0.01_{\mathrm{b}}\end{array}$ & $\begin{array}{c}\mathrm{B} 10.60 \pm \\
0.01_{\mathrm{bc}}\end{array}$ & Spoiled & - & - & - & - & - \\
\hline $\mathbf{T}_{3}$ & $\begin{array}{c}10.66 \pm \\
0.01_{a}\end{array}$ & $\begin{array}{c}10.65 \pm \\
0.01_{\mathrm{a}}\end{array}$ & $\begin{array}{c}\mathrm{AC}^{10.64 \pm} \\
0.01_{\mathrm{a}}\end{array}$ & $\begin{array}{c}{ }^{A C} 10.64 \pm \\
0.01_{a}\end{array}$ & $\begin{array}{c}10.61 \pm \\
0.01_{b}\end{array}$ & $\begin{array}{c}{ }^{\mathrm{B}} 10.55 \pm \\
0.02_{\mathrm{cd}}\end{array}$ & $\begin{array}{c}{ }^{\mathrm{B}} 10.54 \pm \\
0.02_{\mathrm{de}}\end{array}$ & $\begin{array}{c}{ }^{\mathrm{B}} 10.52 \pm \\
0.01_{\mathrm{e}}\end{array}$ & $\begin{array}{c}{ }^{\mathrm{B}} 10.48 \pm \\
0.01_{\mathrm{f}}\end{array}$ & $\begin{array}{c}{ }^{\mathrm{B}} 10.59 \pm \\
0.01\end{array}$ \\
\hline Days mean & $\begin{array}{c}10.66 \pm \\
0.01_{\mathrm{a}}\end{array}$ & $\begin{array}{c}10.64 \pm \\
0.01_{\mathrm{ab}}\end{array}$ & $\begin{array}{c}10.63 \pm \\
0.01_{b}\end{array}$ & $\begin{array}{c}10.62 \pm \\
0.01_{\mathrm{bc}}\end{array}$ & - & - & - & - & - & \\
\hline \multicolumn{11}{|c|}{ pH } \\
\hline $\mathbf{T}_{1}$ & $\begin{array}{l}5.98 \pm \\
0.01_{a}\end{array}$ & $\begin{array}{l}{ }^{\mathrm{A}} 6.02 \pm \\
0.01_{\mathrm{b}}\end{array}$ & $\begin{array}{c}\mathrm{AC}_{5.99 \pm} \\
0.01_{\mathrm{a}}\end{array}$ & $\begin{array}{c}{ }^{\mathrm{AC}} 6.02 \pm \\
0.01_{\mathrm{bc}}\end{array}$ & $\begin{array}{l}{ }^{\mathrm{A}} 6.09 \pm \\
0.01_{\mathrm{d}}\end{array}$ & $\begin{array}{l}{ }^{\mathrm{A}} 6.17 \pm \\
0.01_{\mathrm{e}}\end{array}$ & $\begin{array}{l}{ }^{\mathrm{A}} 6.21_{ \pm} \\
0.01_{\mathrm{f}}\end{array}$ & $\begin{array}{l}{ }^{\mathrm{A}} 6.21_{ \pm} \\
0.01_{\mathrm{g}}\end{array}$ & $\begin{array}{l}\text { A } 6.25 \pm \\
0.01_{\mathrm{h}}\end{array}$ & $\begin{array}{l}{ }^{\mathrm{A}} 6.10 \pm \\
0.01\end{array}$ \\
\hline $\mathrm{T}_{2}$ & $\begin{array}{l}5.98 \pm \\
0.01_{a}\end{array}$ & $\begin{array}{l}\text { B } 6.04 \pm \\
0.01_{b}\end{array}$ & $\begin{array}{l}{ }^{\mathrm{B}} 6.16 \pm \\
0.01_{\mathrm{c}}\end{array}$ & $\begin{array}{l}{ }^{\mathrm{B}} 6.31 \pm \\
0.01_{\mathrm{d}}\end{array}$ & Spoiled & - & - & - & - & - \\
\hline $\mathbf{T}_{3}$ & $\begin{array}{c}5.98 \pm \\
0.01_{\mathrm{a}}\end{array}$ & $\begin{array}{c}\mathrm{C}_{5.99 \pm} \\
0.01_{\mathrm{a}}\end{array}$ & $\begin{array}{c}\mathrm{C}_{5.99 \pm} \\
0.01_{\mathrm{a}}\end{array}$ & $\begin{array}{l}\mathrm{C}_{6.01 \pm} \\
0.01_{\mathrm{b}}\end{array}$ & $\begin{array}{l}\mathrm{B} 6.05 \pm \\
0.01_{\mathrm{c}}\end{array}$ & $\begin{array}{l}{ }^{\mathrm{B}} 6.10 \pm \\
0.01_{\mathrm{d}}\end{array}$ & $\begin{array}{l}{ }^{\mathrm{B}} 6.12 \pm \\
0.01_{\mathrm{e}}\end{array}$ & $\begin{array}{l}{ }^{\mathrm{B}} 6.14 \pm \\
0.01_{\mathrm{f}}\end{array}$ & $\begin{array}{l}{ }^{\mathrm{B}} 6.19 \pm \\
0.01_{\mathrm{g}}\end{array}$ & $\begin{array}{l}{ }^{\mathrm{B}} 6.06 \pm \\
0.01\end{array}$ \\
\hline Days mean & $\begin{array}{l}5.98 \pm \\
0.01_{\mathrm{a}}\end{array}$ & $\begin{array}{l}6.02 \pm \\
0.01_{b}\end{array}$ & $\begin{array}{l}6.05 \pm \\
0.02_{c}\end{array}$ & $\begin{array}{l}6.11 \pm \\
0.03_{d}\end{array}$ & - & - & - & - & - & \\
\hline
\end{tabular}


Int.J.Curr.Microbiol.App.Sci (2018) 7(12): 3154-3164

\begin{tabular}{|c|c|c|c|c|c|c|c|c|c|c|}
\hline \multirow{3}{*}{$\begin{array}{c}\text { Treatmen } \\
\mathbf{t}\end{array}$} & \multicolumn{9}{|c|}{ Storage period (Days) } & \multirow{3}{*}{$\begin{array}{c}\text { Treat- } \\
\text { ment } \\
\text { mean }\end{array}$} \\
\hline & $\mathbf{0}$ & 4 & 8 & 12 & 16 & 20 & 24 & 28 & 32 & \\
\hline & \multicolumn{9}{|c|}{ TBA (mg malanoaldehyde/kg) } & \\
\hline $\mathbf{T}_{1}$ & $\begin{array}{c}0.30 \pm \\
0.01_{\mathrm{a}}\end{array}$ & $\begin{array}{c}{ }^{\mathrm{AC}} 0.32 \pm \\
0.01_{\mathrm{ab}}\end{array}$ & $\begin{array}{c}{ }^{\mathrm{AC}} 0.34 \pm \\
0.01_{b}\end{array}$ & $\begin{array}{c}{ }^{\mathrm{AC}} 0.37 \pm \\
0.01_{\mathrm{c}}\end{array}$ & $\begin{array}{l}0.39 \pm \\
0.01_{\mathrm{cd}}\end{array}$ & $\begin{array}{c}0.44 \pm \\
0.01_{e}\end{array}$ & $\begin{array}{c}0.48 \pm \\
0.01_{f}\end{array}$ & $\begin{array}{l}{ }^{\mathrm{A}} 0.55 \pm \\
0.02_{\mathrm{g}}\end{array}$ & $\begin{array}{l}{ }^{\mathrm{A}} 0.62 \pm \\
0.01_{\mathrm{h}}\end{array}$ & $\begin{array}{c}0.42 \pm \\
0.01\end{array}$ \\
\hline $\mathbf{T}_{2}$ & $\begin{array}{c}0.30 \pm \\
0.01_{\mathrm{a}}\end{array}$ & $\begin{array}{c}{ }^{\mathrm{B}} 0.39 \pm \\
0.01_{\mathrm{b}}\end{array}$ & $\begin{array}{c}{ }^{\mathrm{B}} 0.59 \pm \\
0.01_{\mathrm{c}}\end{array}$ & $\begin{array}{c}{ }^{\mathrm{B}} 0.66 \pm \\
0.01_{\mathrm{d}}\end{array}$ & Spoiled & - & - & - & - & - \\
\hline $\mathbf{T}_{3}$ & $\begin{array}{c}0.30 \pm \\
0.01_{\mathrm{a}}\end{array}$ & $\begin{array}{l}{ }^{\mathrm{C}} 0.32 \pm \\
0.01_{\mathrm{ab}}\end{array}$ & $\begin{array}{l}\mathrm{C}_{0.33 \pm} \\
0.01_{\mathrm{b}}\end{array}$ & $\begin{array}{c}\mathrm{C}_{0.38 \pm} \\
0.01_{\mathrm{c}}\end{array}$ & $\begin{array}{l}0.38 \pm \\
0.01_{\mathrm{cd}}\end{array}$ & $\begin{array}{c}0.42 \pm \\
0.01_{e}\end{array}$ & $\begin{array}{c}0.47 \pm \\
0.01_{f}\end{array}$ & $\begin{array}{l}{ }^{\mathrm{B}} 0.52 \pm \\
0.01_{\mathrm{g}}\end{array}$ & $\begin{array}{l}{ }^{\mathrm{B}} 0.58 \pm \\
0.01_{\mathrm{h}}\end{array}$ & $\begin{array}{c}0.41 \pm \\
0.01\end{array}$ \\
\hline $\begin{array}{l}\text { Days } \\
\text { mean } \\
\end{array}$ & $\begin{array}{c}0.30 \pm \\
0.01_{\mathrm{a}}\end{array}$ & $\begin{array}{l}0.34 \pm \\
0.01_{b}\end{array}$ & $\begin{array}{l}0.42 \pm \\
0.03_{c}\end{array}$ & $\begin{array}{l}0.47 \pm \\
0.03_{\mathrm{d}}\end{array}$ & - & - & - & - & - & \\
\hline \multicolumn{11}{|c|}{ Tyrosine $(\mathrm{mg} / \mathbf{1 0 0 g})$} \\
\hline $\mathbf{T}_{1}$ & $\begin{array}{c}11.29 \pm \\
0.31_{\mathrm{a}}\end{array}$ & $\begin{array}{c}{ }^{\mathrm{AC}_{1}} 12.21 \pm \\
0.28_{\mathrm{a}}\end{array}$ & $\begin{array}{c}{ }^{{ }^{A C}} 13.42 \pm \\
0.21_{b}\end{array}$ & $\begin{array}{c}{ }^{\mathrm{AC}} 14.25 \pm \\
0.20_{\mathrm{c}}\end{array}$ & $\begin{array}{c}{ }^{\mathrm{A}} 14.96 \pm \\
0.12_{\mathrm{d}}\end{array}$ & $\begin{array}{c}\mathrm{A}_{15.75 \pm} \\
0.37 \mathrm{e}\end{array}$ & $\begin{array}{c}\mathrm{A}_{16.88 \pm} \\
0.33_{\mathrm{f}}\end{array}$ & $\begin{array}{c}\mathrm{A}_{18.83 \pm} \\
0.41_{\mathrm{g}}\end{array}$ & $\begin{array}{c}\mathrm{A}_{23.21 \pm} \\
0.78_{\mathrm{h}}\end{array}$ & $\begin{array}{c}\mathrm{A}_{15.64 \pm} \\
0.48\end{array}$ \\
\hline $\mathbf{T}_{2}$ & $\begin{array}{c}11.29 \pm \\
0.31_{\mathrm{a}}\end{array}$ & $\begin{array}{c}{ }^{\mathrm{B}} 14.13 \pm \\
0.58_{\mathrm{b}}\end{array}$ & $\begin{array}{c}{ }^{\mathrm{B}} 18.54 \pm \\
0.47_{\mathrm{c}}\end{array}$ & $\begin{array}{c}\text { B } 24.42 \pm \\
0.62_{d}\end{array}$ & Spoiled & - & - & - & - & - \\
\hline $\mathbf{T}_{3}$ & $\begin{array}{c}11.29 \pm \\
0.31_{\mathrm{a}}\end{array}$ & $\begin{array}{c}\mathrm{C}_{11.38 \pm} \\
0.42_{\mathrm{ab}}\end{array}$ & $\begin{array}{c}\mathrm{C}_{12.08 \pm} \\
0.30_{\mathrm{a}}\end{array}$ & $\begin{array}{c}{ }^{C} 12.38 \pm \\
0.46_{b}\end{array}$ & $\begin{array}{c}{ }^{\mathrm{B}} 12.92 \pm \\
0.46_{\mathrm{c}}\end{array}$ & $\begin{array}{c}{ }^{\mathrm{B}} 13.04 \pm \\
0.46_{\mathrm{d}}\end{array}$ & $\begin{array}{c}{ }^{\mathrm{B}} 14.25 \pm \\
0.50_{\mathrm{e}}\end{array}$ & $\begin{array}{c}\mathrm{B} 14.75 \pm \\
0.40_{\mathrm{f}}\end{array}$ & $\begin{array}{c}\mathrm{B}_{16.54 \pm} \\
0.68_{\mathrm{g}}\end{array}$ & $\begin{array}{c}\mathrm{B}_{13.18 \pm} \\
0.26\end{array}$ \\
\hline $\begin{array}{l}\text { Days } \\
\text { mean }\end{array}$ & $\begin{array}{c}11.29 \pm \\
0.17_{\mathrm{a}}\end{array}$ & $\begin{array}{c}12.57 \pm \\
0.37_{b}\end{array}$ & $\begin{array}{c}14.68 \pm \\
0.70_{c}\end{array}$ & $\begin{array}{c}17.01 \pm \\
1.31_{\mathrm{d}}\end{array}$ & - & - & - & - & - & \\
\hline
\end{tabular}

Values are Mean \pm S.E. of three replications $(n=6)$

Means \pm S.E with different superscripts in a column (Capital letters) or row (Small letters) differ significantly $(\mathrm{P}<0.05)$.

Table. 2 Microbiological changes in aerobically packed superchilled chicken momos during storage

\begin{tabular}{|c|c|c|c|c|c|c|c|c|c|c|}
\hline \multirow{3}{*}{ Treatment } & \multicolumn{9}{|c|}{ Storage period (Days) } & \multirow{3}{*}{$\begin{array}{l}\text { Treatment } \\
\text { mean }\end{array}$} \\
\hline & $\mathbf{0}$ & 4 & 8 & 12 & 16 & 20 & 24 & 28 & 32 & \\
\hline & \multicolumn{9}{|c|}{ TPC ( $\left.\log _{10} \mathrm{cfu} / \mathrm{g}\right)$} & \\
\hline $\mathbf{T}_{1}$ & $\begin{array}{l}1.55 \pm \\
0.69_{\mathrm{a}}\end{array}$ & $\begin{array}{c}{ }^{\mathrm{AC}} 2.68 \pm \\
0.54 \mathrm{~b}\end{array}$ & $\begin{array}{c}{ }^{\mathrm{AC}} 3.30 \pm \\
0.11_{\mathrm{bc}}\end{array}$ & $\begin{array}{c}\mathrm{AC}_{3.70 \pm} \\
0.16_{\mathrm{cd}}\end{array}$ & $\begin{array}{l}3.92 \pm \\
0.13_{\mathrm{de}}\end{array}$ & $\begin{array}{l}4.14 \pm \\
0.13_{\text {ef }}\end{array}$ & $\begin{array}{l}4.37 \pm \\
0.11_{\mathrm{fg}}\end{array}$ & $\begin{array}{l}4.57 \pm \\
0.05_{\text {ghi }}\end{array}$ & $\begin{array}{c}4.83 \pm \\
0.10_{i}\end{array}$ & $\begin{array}{l}\text { A } 3.67 \pm \\
0.16\end{array}$ \\
\hline $\mathbf{T}_{2}$ & $1.55 \pm 0.69 \mathrm{a}$ & ${ }^{\mathrm{B}} 3.59 \pm 0.08_{\mathrm{b}}$ & ${ }^{\mathrm{B}} 4.58 \pm 0.05_{\mathrm{c}}$ & ${ }^{\mathrm{B}} 5.02 \pm 0.06_{\mathrm{cd}}$ & Spoiled & - & - & - & - & - \\
\hline $\mathbf{T}_{3}$ & $\begin{array}{l}1.55 \pm \\
0.69 \mathrm{a}\end{array}$ & $\begin{array}{c}\mathrm{C}_{2.10 \pm} \\
0.67_{\mathrm{ab}}\end{array}$ & $\begin{array}{l}\mathrm{C}_{2.72 \pm} \\
0.55_{\mathrm{b}}\end{array}$ & $\begin{array}{l}\mathrm{C}_{3.10 \pm} \\
0.06_{\mathrm{bc}}\end{array}$ & $\begin{array}{l}3.57 \pm \\
0.20_{\mathrm{cd}}\end{array}$ & $\begin{array}{l}3.77 \pm \\
0.08_{\mathrm{de}}\end{array}$ & $\begin{array}{l}4.03 \pm \\
0.13_{\text {ef }}\end{array}$ & $\begin{array}{l}4.36 \pm \\
0.11_{\mathrm{gh}}\end{array}$ & $\begin{array}{l}4.47 \pm \\
0.10_{\mathrm{h}}\end{array}$ & $\begin{array}{l}{ }^{\mathrm{B}} 3.30 \pm \\
0.18\end{array}$ \\
\hline Days mean & $1.55 \pm 0.38_{a}$ & $2.79 \pm 0.31_{b}$ & $3.53 \pm 0.26_{c}$ & $3.94 \pm 0.20_{\mathrm{d}}$ & - & - & - & - & - & \\
\hline \multicolumn{11}{|c|}{ PPC (Log $10 \mathrm{cfu} / \mathrm{g})$} \\
\hline $\mathbf{T}_{1}$ & ND & ND & ND & ND & ND & $\begin{array}{c}2.68 \pm \\
0.54_{a}\end{array}$ & $\begin{array}{l}\mathrm{A}_{3.50 \pm} \\
0.07_{\mathrm{a}}\end{array}$ & $\begin{array}{l}\mathrm{A}_{3.53 \pm} \\
0.06_{\mathrm{bc}}\end{array}$ & $\begin{array}{l}3.69 \pm \\
0.03_{\mathrm{cd}}\end{array}$ & $\begin{array}{l}{ }^{\mathrm{A}} 1.49 \pm \\
0.24\end{array}$ \\
\hline $\mathbf{T}_{2}$ & ND & ND & $\begin{array}{c}3.25 \pm \\
0.10\end{array}$ & $\begin{array}{l}3.71 \pm \\
0.05\end{array}$ & Spoiled & - & - & - & - & - \\
\hline $\mathbf{T}_{3}$ & ND & ND & ND & ND & ND & ND & $\begin{array}{c}{ }^{\mathrm{B}} 2.60 \pm \\
0.52_{\mathrm{a}}\end{array}$ & $\begin{array}{c}\mathrm{B} 2.65 \pm \\
0.53_{\mathrm{a}}\end{array}$ & $\begin{array}{l}3.46 \pm \\
0.06_{b}\end{array}$ & $\begin{array}{c}{ }^{\mathrm{B}} 0.97 \pm \\
0.20\end{array}$ \\
\hline Days mean & ND & ND & $1.08 \pm 0.37$ & $1.24 \pm 0.42$ & - & - & - & - & - & \\
\hline & \multicolumn{10}{|c|}{ Yeast and mold count $\left(\log _{10} \mathrm{cfu} / \mathrm{g}\right)$} \\
\hline $\mathbf{T}_{1}$ & ND & ND & ND & ND & ND & ND & $\begin{array}{r}0.95 \pm \\
0.60_{\mathrm{a}}\end{array}$ & $\begin{array}{l}1.90 \pm \\
0.60_{\mathrm{bc}}\end{array}$ & $\begin{array}{l}2.03 \pm \\
0.65_{c}\end{array}$ & $\begin{array}{c}0.54 \pm \\
0.16\end{array}$ \\
\hline $\mathbf{T}_{2}$ & ND & $\begin{array}{l}1.40 \pm \\
0.63_{\mathrm{a}}\end{array}$ & $\begin{array}{l}1.93 \pm \\
0.61_{\mathrm{ab}}\end{array}$ & $\begin{array}{c}2.68 \pm \\
0.54_{b}\end{array}$ & spoiled & - & - & - & - & - \\
\hline $\mathbf{T}_{3}$ & ND & ND & ND & ND & ND & ND & ND & ND & ND & 0.00 \\
\hline
\end{tabular}

Values are Mean \pm S.E. of three replications $(n=6)$

Means \pm S.E with different superscripts in a column (Capital letters) or row (Small letters) differ significantly $(\mathrm{P}<0.05)$.

$\mathrm{ND}=$ Not Detected 
Table.3 Sensory changes in aerobically packed superchilled chicken momos during storage

\begin{tabular}{|c|c|c|c|c|c|c|c|c|c|}
\hline \multirow[b]{2}{*}{ Treatment } & \multicolumn{8}{|c|}{ Storage period (Days) } & \multirow{2}{*}{$\begin{array}{l}\text { Treatment } \\
\text { mean }\end{array}$} \\
\hline & $\mathbf{0}$ & 4 & 8 & 12 & 16 & 20 & 24 & 28 & \\
\hline \multicolumn{10}{|c|}{ Overall palatability } \\
\hline$\overline{T_{1}}$ & $\begin{array}{l}7.57 \pm \\
0.12_{a}\end{array}$ & $\begin{array}{c}{ }^{\mathrm{AB}} 6.77 \pm \\
0.12_{\mathrm{bg}}\end{array}$ & $\begin{array}{c}{ }^{\mathrm{AC}} 6.77 \pm \\
0.12_{\text {bgcf }}\end{array}$ & $\begin{array}{l}6.53 \pm \\
0.23_{b d}\end{array}$ & $\begin{array}{c}6.67 \pm \\
0.12_{\text {befg }}\end{array}$ & $\begin{array}{c}6.40 \pm \\
0.17_{\text {fdh }}\end{array}$ & $\begin{array}{l}{ }^{\mathrm{A}} 6.93 \pm \\
0.15_{\mathrm{ge}}\end{array}$ & $\begin{array}{l}6.23 \pm \\
0.21_{\text {hd }}\end{array}$ & $\begin{array}{c}6.73 \pm \\
0.06\end{array}$ \\
\hline $\mathbf{T}_{2}$ & $\begin{array}{l}7.57 \pm \\
0.12_{a}\end{array}$ & $\begin{array}{c}{ }^{\mathrm{A}} 6.40 \pm \\
0.14_{\mathrm{bc}}\end{array}$ & $\begin{array}{c}{ }^{\mathrm{B}} 6.13 \pm \\
0.24_{\mathrm{c}}\end{array}$ & Spoiled & - & - & - & - & - \\
\hline $\mathbf{T}_{3}$ & $\begin{array}{l}7.57 \pm \\
0.12_{a}\end{array}$ & $\begin{array}{c}{ }^{\mathrm{B}} 6.90 \pm \\
0.13_{b}\end{array}$ & $\begin{array}{c}{ }^{c} 6.90 \pm \\
0.16_{c}\end{array}$ & $\begin{array}{l}6.53 \pm \\
0.23_{\mathrm{dc}}\end{array}$ & $\begin{array}{l}6.87 \pm \\
0.13_{\mathrm{ec}}\end{array}$ & $\begin{array}{c}6.60 \pm \\
0.11_{\text {fcg }}\end{array}$ & $\begin{array}{l}{ }^{\mathrm{B}} 6.40 \pm \\
0.13_{\mathrm{gdh}}\end{array}$ & $\begin{array}{c}6.38 \pm \\
0.20_{\text {hdf }}\end{array}$ & $\begin{array}{c}6.77 \pm \\
0.06\end{array}$ \\
\hline $\begin{array}{l}\text { Days } \\
\text { mean }\end{array}$ & $\begin{array}{l}7.57 \pm \\
0.07 \mathrm{a}\end{array}$ & $\begin{array}{l}6.69 \pm \\
0.08_{\mathrm{bc}}\end{array}$ & $\begin{array}{c}6.60 \pm \\
0.11_{c}\end{array}$ & - & - & - & - & - & \\
\hline
\end{tabular}

\begin{tabular}{|c|c|c|c|c|c|c|c|c|c|c|c|}
\hline & \multicolumn{7}{|c|}{ Storage period (Days) } \\
Treat- \\
ment
\end{tabular}




\begin{tabular}{|c|c|c|c|c|c|c|c|c|c|}
\hline $\mathbf{T}_{3}$ & $\begin{array}{l}7.17 \pm \\
0.19 a\end{array}$ & $\begin{array}{l}{ }^{\mathrm{C}} 6.63 \pm \\
0.16_{\mathrm{bd}}\end{array}$ & $\begin{array}{l}{ }^{\mathrm{C}} 6.43 \pm \\
0.28_{\mathrm{bc}}\end{array}$ & $\begin{array}{l}{ }^{\mathrm{B}} 6.70 \pm \\
0.15_{\mathrm{dce}}\end{array}$ & $\begin{array}{l}{ }^{\mathrm{B}} 6.63 \pm \\
0.09_{\text {bef }}\end{array}$ & $\begin{array}{l}{ }^{\mathrm{B}} 6.60 \pm \\
0.16_{\text {bfd }}\end{array}$ & $\begin{array}{l}{ }^{\mathrm{B}} 5.77 \pm \\
0.16_{\mathrm{g}}\end{array}$ & $\begin{array}{l}{ }^{\mathrm{B}} 6.07 \pm \\
0.16_{\mathrm{hc}}\end{array}$ & $\begin{array}{l}{ }^{\mathrm{B}} 6.50 \pm \\
0.07\end{array}$ \\
\hline $\begin{array}{l}\text { Days } \\
\text { mean }\end{array}$ & $\begin{array}{l}7.17 \pm \\
0.11_{a}\end{array}$ & $\begin{array}{l}6.78 \pm \\
0.10_{b}\end{array}$ & $\begin{array}{l}6.71 \pm \\
0.15_{c}\end{array}$ & - & - & - & - & - & \\
\hline \multicolumn{10}{|c|}{ Texture } \\
\hline $\mathbf{T}$ & $\begin{array}{l}7.27 \pm \\
0.14_{\mathrm{ab}}\end{array}$ & $\begin{array}{c}{ }^{\mathrm{AC}} 6.90 \pm \\
0.11_{\mathrm{a}}\end{array}$ & $\begin{array}{l}{ }^{\mathrm{A}} 6.87 \pm \\
0.10_{\mathrm{bc}}\end{array}$ & $\begin{array}{l}6.43 \pm \\
0.19_{d}\end{array}$ & $\begin{array}{r}6.43 \pm \\
0.13_{\mathrm{d}}\end{array}$ & $\begin{array}{l}{ }^{\mathrm{A}} 6.37 \pm \\
0.16_{\mathrm{de}}\end{array}$ & $\begin{array}{l}6.57 \pm \\
0.20_{\mathrm{df}}\end{array}$ & $\begin{array}{l}6.23 \pm \\
0.17_{\mathrm{dg}}\end{array}$ & $\begin{array}{c}6.63 \pm \\
0.06\end{array}$ \\
\hline $\mathbf{T}_{2}$ & $\begin{array}{l}7.27 \pm \\
0.14_{a}\end{array}$ & $\begin{array}{c}\mathrm{B} 6.33 \pm \\
0.15_{\mathrm{b}}\end{array}$ & $\begin{array}{c}\mathrm{B} 6.07 \pm \\
0.19_{\mathrm{bc}}\end{array}$ & Spoiled & - & - & - & - & - \\
\hline $\mathbf{T}_{3}$ & $\begin{array}{l}7.27 \pm \\
0.14_{\mathrm{ac}}\end{array}$ & $\begin{array}{l}{ }^{\mathrm{C}} 6.83 \pm \\
0.15_{\mathrm{be}}\end{array}$ & $\begin{array}{l}{ }^{\mathrm{C}} 6.37 \pm \\
0.23_{\mathrm{cdg}}\end{array}$ & $\begin{array}{l}6.43 \pm \\
0.23_{d}\end{array}$ & $\begin{array}{c}6.37 \pm \\
0.14_{\text {cdg }}\end{array}$ & $\begin{array}{l}{ }^{\mathrm{B}} 6.87 \pm \\
0.12_{\mathrm{e}}\end{array}$ & $\begin{array}{l}6.23 \pm \\
0.15_{\mathrm{fd}}\end{array}$ & $\begin{array}{l}6.40 \pm \\
0.16_{\mathrm{dg}}\end{array}$ & $\begin{array}{c}6.60 \pm \\
0.07\end{array}$ \\
\hline $\begin{array}{l}\text { Days } \\
\text { mean }\end{array}$ & $\begin{array}{l}7.27 \pm \\
0.08_{a}\end{array}$ & $\begin{array}{l}6.69 \pm \\
0.09_{b}\end{array}$ & $\begin{array}{l}6.43 \pm \\
0.11_{c}\end{array}$ & - & - & - & - & - & \\
\hline
\end{tabular}

Values are Mean \pm S.E. of three replications $(n=15)$

Means \pm S.E with different superscripts in a column (Capital letters) or row (Small letters) differ significantly $(\mathrm{P}<$ $0.05)$.

Thereafter TBA values in superchilling and frozen samples increased consistently throughout the storage period. However, this increase was significantly higher in superchilled samples as compared to frozen samples. The increase in TBA value during storage period was mainly attributed to the oxygen permeability of packaging material (Sen, 1996) and/or due to lipid oxidation (Strange et al., 1977). Present findings were in agreement with the findings of Vanramhlimpuii (2015) in chicken momo stored at $4 \pm 1^{\circ} \mathrm{C}$ and Kanle (2017) for superchilled nuggets.

Similar pattern was also observed in tyrosine value of chilled, superchilled and frozen samples. The tyrosine value was significantly lower in frozen sample than superchilled samples throughout the study. However, it was consistently highest in chilled samples upto day 12 which might be due to intrinsic (autolysis) changes in chicken momo and bacterial action (Agnihotri, 1988; Dainty et al., 1975; Strange et.al., 1977). The findings of Vanramhlimpuii (2015) in chicken momo stored at $4 \pm 1^{\circ} \mathrm{C}$ and Kanle (2017) for superchilled nuggets also supported the present results.

\section{Microbiological analysis}

There was gradual increase in total plate count (TPC) of all the samples during entire storage period (Table 2). However, superchilled and frozen samples had significantly lower TPC than chilled samples upto day 12. This reduction in TPC of superchilled and frozen samples could be attributed to the temperature effect due to which microbes became dormant and did not multiply (Leygonie et al., 2012) and in both the samples damage caused to the cells through the formation of ice crystals (Warris, 2010). A linear increase in total plate counts of chicken nuggets stored under superchilled and frozen conditions was also reported by Kanle (2017).

The Psychrophillic count (PPC) in chicken momos were absent in superchilled and frozen samples upto 16 days and in chilled samples upto 4 days. The PPC were recorded in superchilled sample on day 20 and onwards, which was significantly higher than frozen samples. This increase in Psychrophillic count could be due to increased enzymatic activity of psychrotrophs at low temperature which might have contributed to deterioration of 
meat quality (Kandeepan and Biswas, 2007). Similar trends were also reported by Rathod (2017) and Kanle (2017) for superchilled breast fillets and chicken nuggets respectively. The yeast and molds were not detected in chicken momos on day 0 . However it was gradually increased in chilled sample from day 4 onwards while superchilled samples had the yeast and mold on day 24 onwards. This might be due to post processing contamination and handling. Das et al., (2013) and Singh et al., (2011) also reported similar results in chicken nuggets and chicken snacks, respectively. However, frozen sample did not reveal any yeast and mold count. Sutherland et al., (1975) and Babji and Murthy (2000) also recorded very low yeast and mould counts during storage of beef and for minced goat meat at low temperature respectively.

Further, none of the samples revealed coliforms in chicken momos throughout the period of storage. It could be either due to the destruction of these bacteria during cooking at high temperature, much above their death point of $57^{\circ} \mathrm{C}$ or no contamination during post processing handling of chicken momos. Tanuja (2013) also reported similar findings who reported no coliform count for the chicken momos throughout storage study at chilling temperature. Similar results were also reported by Rathod (2017) in chicken breast fillets at superchilling temperature.

\section{Sensory scores}

It was observed that the sensory scores of appearance, juiciness and overall palatability were higher for superchilled samples than frozen samples throughout the study (Table 3). However overall palatability scores of superchilled samples significantly reduced on day 28 and the sample was subsequently spoiled on day 32 whereas chilled samples were spoiled on day 12 . The sensory score of all samples were decreased gradually during entire storage period. Moreover, there was no significant variation in appearance of chicken momo in all treatments. The flavor scores increased marginally in superchilled samples during initial storage which were then reduced gradually by the end of storage. However this decrease in flavor scores of superchilled sample was non significant as compared to frozen sample. The juiciness and texture scores of superchilled samples were improved indicating beneficial effect of superchilling in stored products. In case of frozen product, juiciness was reduced significantly during storage period as compared to superchilled samples. Kanle (2017) also reported improvement in juiciness of chicken nuggets when stored under superchilling $\quad\left(2 \pm 0.5^{\circ} \mathrm{C}\right) \quad$ temperature. Similarly, Olafsdottir et al., (2006) also reported improvement in juiciness in superchilled cod during storage.

This improvement in overall palatability of superchilled sample was improved which could be due to improved juiciness and texture of the product. Similar results were also reported by Kanle (2017) in chicken nuggets and Olafsdottir et al., (2006) in superchilled whole cod. Thus it was concluded that the chicken momos superchilled at $-2 \pm 0.5^{\circ} \mathrm{C}$ could be stored conveniently up to 28 days under aerobic conditions.

\section{References}

AOAC, 2012. Official methods of analysis, $16^{\text {th }}$ edition.Association of Official Agriculture Chemists, Washington, W.C.

APHA, 1984. Compendium of methods for microbiological examination of food. Speck, M.L. (ed.) American Public Health Association, Washington, W.C.

Agnihotri, M.K., 1988. A comparative study 
on shelf life and microbial spoilage of refrigerated buffalo meat. Ph.D. Thesis submitted to Indian Veterinary Research Institute, Izatnagar.

Babji, Y. and T. R. K. Murthy., 2000. Effect of inoculation of mesophilic lactic acid bacteria on microbial and sensory changes of minced goat meat during storage under vacuum and subsequent aerobic storage. Meat Sci. 54:197-202.

Dainty, R.H., B. G. Shaw, K. A. Boer and E.S.J. Scheps (1975) Protein changes caused by bacterial growth in beef. $\mathrm{J}$. Applied Bacteriology, 39: 73-81.

Das, A., D.R. Nath, S. Kumari and R. Saha, 2013. Effect of fermented bamboo shoot on the quality and shelf life of nuggets prepared from desi spent hen. Vet World 6 (7):419-423

Duun, A.S., A.K.T. Hemmingsen, A. Haugland and T. Rustad, 2008. Quality changes during superchilled storage of pork roast. Food Sci. and Technol. 41 (10): 2136-2143.

Duun, A. S., and T. Rustad, 2007. Quality changes during superchilled storage of cod (Gadus morhua) fillets. Food Chem. 105(3): 1067-1075.

Duun, A. S., and T. Rustad, 2008. Quality of superchilled vacuum packed Atlantic salmon (Salmosalar) fillets stored at 1.4 and $-3.6^{\circ} \mathrm{C}$. Food Chem. 106 (1):122-131.

Einarsson, H., 1988. Deep chilling (superchilling, partial freezing) - A literature survey. In SIKs service series (pp.30).Gothenburg, Sweden: SIK The Swedish food institute, Chalmers University of Technology.

Erikson, U., E. Mismi and L. Gallart-Jorne, 2011. Superchilling of rested Atlantic salmon: Different chilling strategies and effects on fish and filet quality. Food Chem.127:1427-1437.

Fennema, O.R., 1973. Nature of freezing process. In: O.R. Fennema, W.D.
Powrie and E.H. Marth (Eds.), Low temperature preservation of foods and living matter (pp. 151-227). New York: Marcel Dekker.

Hiwarkar P.P., 2017. Development and quality assessment of meat based novel product: chicken chakali. M.V.Sc. thesis submitted to Maharashtra Animal and Fishery Sciences University, Nagpur.

Huff-Lonergan, E. and S.M. Lonergan, 2005. Mechanisms of water-holding capacity of meat: The role of post mortem biochemical and structural changes. Meat Sci. 71:194-204.

Hemmingsen, A.K.T., 2002. Quality of fresh foods. PhD Thesis. Faculty of Engineering Science and Technology, Department of Energy and Process Engineering (pp. IX, 26, [86]). Trondheim: Norwegian University of Science and Technology (NTNU)

Kandeepan, G. and S. Biswas, 2007. Effect of domestic refrigeration on keeping quality of buffalo meat. J. Food Technol. 5 (1): 29-35.

Kaale, L.D., T.M. Eikevik, T. Bardel, E. Kjorsvik and T. S. Nordtvedt, 2013. The effect of cooling rates on the ice crystal growth in air-packed salmon fillets during superchilling and superchilled storage. Int. J.Refri. 36: 110-119.

Kanle, P.C., 2017. Effect of super chilling on the shelf life of chicken nuggets. M.V.Sc. thesis submitted to Maharashtra Animal and Fishery Sciences University, Nagpur

Keeton, J. T., 1983. Effect of fat and $\mathrm{NaCl} /$ phosphate levels on the chemical and sensory properties of pork patties. J. Food sci. 48: 878-881.

Leygonie, C., T. J. Britz and L.C. Hoffman, 2012. Impact of freezing and thawing on the quality of meat: Review. Meat Sci., 91(2), 93-98.

Magnussen, O.M., A. Haugland, A.K. 
Torstveit, S. Johansen, S. and T.S. Nordtvedt, 2008. Advances in superchilling of food-Process characteristics and product quality. Trends Food Sci. Technol. 19:418-424.

Mishra, B. P., Geeta Chauhan, Mendiretta S. K., Rath P. K. and Renuka Nayar, 2015. Storage stability of aerobically packaged extended dehydrated chicken meat rings at ambient temperature. Turk. J. Vet Anim. Sci. 39: 493-500 () TÜBİTAK doi:10.3906/vet-1406-93

Olafsdottir, G., H.E.L.E. Lauzon, E.I.A. Martinsdottir, Oehlenschlauger J. and Kristbergsson K., 2006. Evaluation of shelf life of superchilled cod (Gadus morhua) fillets and the influence of temperature fluctuations during storage on microbial and chemical quality indicators. Journal of Food Sci., 71: S97-S109.

Raja, W.H., S. Kumar, Z. F. Bhat and P.Kumar, 2014. Effect of ambient storage on the quality characteristics of aerobically packaged fish curls incorporated with different flours Springer Plus, doi:10.1186/2193-18013-106

Rathod, K.S., R. K. Ambadkar, B.M. Naveena and S. K. Devatkal, 2017 a . Quality analysis of vacuum packaged super chilled chicken breast at 24 hour storage. Int. J. Curr. Microbiol. App. Sci. 6(2): 675-683

Rathod, K.S., R. K. Ambadkar, and B.M. Naveena, $2017^{\mathrm{b}}$. Quality of superchilled poultry meat at storage. J. Meat Sci. 12(1): 39-43

Rathod, K. S., 2017. Effect of packaging and superchilling on the quality and shelf life of chicken. Ph.D. thesis submitted to Maharashtra Animal and Fishery Sciences University, Nagpur

Sen, A.R., 1996. Quality changes in buffalo meat due to freeze-thaw cycles during storage. Ph.D. Thesis submitted to
Indian Veterinary Research Institute, Izatnagar

Sharma, B.D. and P.K. Nanda, 2002. Studies on the development and storage stability of chicken chips, Indian J. poult. Sci, Vol. 37 No. 2, pp. 155-158.

Singh, V. P., M. K. Sanyal, P. C. Dubey, N. Sachan and V. Kumar, 2011. Chicken snacks as affected by storage conditions under aerobic and vacuum packaging at $30 \pm 2^{\circ} \mathrm{C}$. African. J. food sci. 5(11): 620625.

Sivertsvik, M., W.K. Jeksurd and J.T. Rosnes, 2002. A review of modified atmosphere packaging of fish and fishery products significance of microbial growth, activities and safety. Int. J. Sci. Technol., 37: 107-127.

Sivertsvik, M., J.T. Rosnes and G.H. Kleiberg, 2003. Effect of modified atmosphere packaging and superchilled storage on the microbial and sensory quality of Atlantic salmon (Salmosalar) fillets. J. Food Sci.68:1467-1472.

Snedecor, G. W., and W. J. Cochran, 1989. Statistical methods, 8th edition. Iowa State University Press, Amer., Iowa, USA.

Strange, E.D., R.C. Benedict, J. L. Smith and C.E. Swift, 1977. Evaluation of rapid test for monitoring alterations in meat quality during storage. I. Intact Meat. J. Food Prot., 40: 843-847.

Sutherland, J. P., J. T. Patterson and J. G. Murray, 1975.Some metabolic and biochemical characteristics of representative microbial isolates from vacuum packaged beef. J. Appl. Bact. 39:227-237.

Tanuja, 2013, Development and quality assessment of meat momos.M.V.Sc. thesis submitted to U.P.Pt. Deen Dayal Upadhyay Pashu Chikitsa Vigyan Vishwavidyalaya Evam GoAnusandhan (DUVASU), Mathura.

Tanuja, V. Pathak, A. K. Varma, V. 
Rajkumar, M. Goswami and V.P. Singh, 2014. Enhancement of quality parameters of chicken momos by incorporation of minced fish meat. J. Meat. Sci., 9 (2): 18-23.

Vanramhlimpuii, 2015. Process standardization of chicken momo. M.V.Sc. thesis submitted to Maharashtra Animal and Fishery Sciences University, Nagpur.

Warris, P. D., 2010. Meat Science - An Introductory Text. 2nd ed. CABI,
Wallingford Oxfordshire, UK.: pp 69 Witte, V. C., G. F. Krause and M. F. Bailey, 1970. A new extraction method for determining 2-thiobarbituric acid values of pork and beef during storage. J. Food Sci.35: 582-585.

Zeng, Q. Z., K. A. Thorarinsdottir and G. Olafsdottir, 2005. Quality changes of shrimp (Pandulus borealis) stored under different cooling conditions. J. Food Sci.70: S459-S466.

\section{How to cite this article:}

Pawade, A.A., K.S. Rathod and Ambadkar, R.K. 2018. Storage Stability of Chicken Momo at Super Chilling Temperature. Int.J.Curr.Microbiol.App.Sci. 7(12): 3154-3164.

doi: https://doi.org/10.20546/ijcmas.2018.712.363 